\title{
Assistência pré-natal pelo Programa Rede Mãe Paranaense em dois municípios de fronteira
}

\author{
Prenatal assistance through the Programa Rede Mãe Paranaense in two border municipalities \\ Assistencia prenatal através del Programa Rede Mãe Paranaense en dos municpios fronterizos
}

Recebido: 09/10/2021 | Revisado: 16/10/2021 | Aceito: 18/10/2021 | Publicado: 19/10/2021

Thaissy Fernanda de Oliveira

ORCID: https://orcid.org/0000-0003-1541-7928

Universidade Estadual do Oeste do Paraná, Brasil

E-mail: thaissy@ hotmail.com

Sebastião Caldeira

ORCID: https://orcid.org/0000-0003-2827-1833

Universidade Estadual do Oeste do Paraná, Brasil

E-mail: calenf3@gmail.com

Letícia Damasceno

ORCID: https://orcid.org/0000-0003-4548-8006

Universidade Estadual do Oeste do Paraná, Brasil

E-mail: letidama09@hotmail.com

Oscar Kenji Nihei

ORCID: https://orcid.org/0000-0002-9156-7787

Universidade Estadual do Oeste do Paraná, Brasil

E-mail: oknihei@gmail.com

Rosangela Aparecida Pimenta Ferrari

ORCID: https://orcid.org/0000-0003-0157-7461

Universidade Estadual de Londrina, Brasil E-mail: ropimentaferrari@uel.br

Adriana Zilly

ORCID: https://orcid.org/0000-0002-8714-8205 Universidade Estadual do Oeste do Paraná, Brasil E-mail: aazilly@ hotmail.com

Ludmila Mourão Xavier

ORCID: https://orcid.org/0000-0001- 6442-5719 Universidade Federal Latino Americana, Brasil E-mail: ludmila.gomes@unila.edu.br

\section{Resumo}

Este estudo buscou apreender a vivência de mulheres que realizaram o pré-natal pelo Programa Rede Mãe Paranaense em dois municípios de faixa de fronteira internacional. Trata-se de uma Pesquisa qualitativa à luz do referencial fenomenológico de Alfred Schütz, realizada com 18 mulheres que foram assistidas no pré-natal pelo referido programa. Para coleta de dados utilizou-se a entrevista fenomenológica e seguiu-se a análise conforme o referencial. Identificaram-se cinco categorias: Conhecimento sobre o Programa; Acesso e acolhimento no serviço de saúde; Atendimento multiprofissional e suas peculiaridades; Educação em saúde e Perspectivas das mulheres frente ao programa. Os resultados apontam para uma prática voltada ao modelo médico-assistencial operada de maneira sistematizada com pouca abertura para diálogos, troca de experiências e informações, considerando as particularidades da área de fronteira e limitação do estudo. Instiga reflexões sobre o cuidado pré-natal diante da proposta do programa que requer investimentos na formação e ações dos profissionais.

Palavras-chave: Cuidado pré-natal; Saúde da mulher; Áreas de fronteira; Planos e programas de saúde; Pesquisa qualitativa.

\footnotetext{
Abstract

This study sought to apprehend the experience of women who underwent prenatal care through the Programa Rede Mãe Paranaense Program in two municipalities on the international border strip. This is a qualitative research in the light of the phenomenological reference of Alfred Schütz, conducted with 18 women who were assisted in prenatal care by the program. For data collection, a phenomenological interview was used, and the analysis was followed according to the referential. Five categories were identified: knowledge about the program; access and reception in the health service; multiprofessional care and its peculiarities; health education; and women's perspectives regarding the program. The results point to a practice focused on the medical care model operated in a systematized way with little opening for dialogues, exchange of experiences and information, considering the particularities of the border area and
} 
the limitation of the study. Instigates reflections on prenatal care in the face of the program proposal that requires investments in training and actions of professionals.

Keywords: Prenatal care; Women's health; Borde áreas; Health plans and programs; Qualitative research.

\section{Resumen}

Este estudio buscó aprehender la experiencia de las mujeres que se sometieron a la atención prenatal a través del Programa Rede Mãe Paranaense en dos municipios del área de la frontera internacional. Se trata de una investigación cualitativa a la luz del marco fenomenológico de Alfred Schütz, realizada con 18 mujeres que fueron asistidas en la atención prenatal por el programa. Para la recogida de datos se utilizó la entrevista fenomenológica y se siguió el análisis según el referencial. Se identificaron cinco categorías: Conocimiento sobre el Programa; Acceso y acolchado en el servicio de salud; Atención multiprofesional y sus peculiaridades; Educación en salud y Perspectivas de las mujeres frente al programa. Los resultados apuntan a una práctica orientada al modelo médico-asistencial operado de forma sistematizada con poca apertura para los diálogos, la búsqueda de experiencias y la información, considerando las particularidades del área de frontera y la limitación del estudio. Insta a reflexionar sobre la atención prenatal ante la propuesta de programa que requiere inversión en formación y acciones de los profesionales.

Palabras clave: Cuidado prenatal; La salud de la mujer; Zonas fronterizas; Planes y programas de salud; Investigación cualitativa.

\section{Introdução}

Este estudo foi desenvolvido em uma região de fronteira que possui algumas peculiaridades, destacando-se a cultura diversificada e a dificuldade de acesso aos serviços de saúde (Gomes, et al., 2019; Mello et al., 2015). No que tange ao atendimento pré-natal, essas particularidades fronteiriças podem interferir de forma significativa na morbimortalidade materna, infantil e neonatal. Por isso, o presente estudo buscou apreender a vivência das mulheres que receberam o cuidado pré-natal pelo Programa Rede Mãe Paranaense (PRMP) em dois municípios situados em região faixa de fronteira internacional no Estado do Paraná (Paraná, 2018).

A taxa de mortalidade materna, infantil e neonatal é uma referência do cuidado no Brasil. Baseado nisso o cuidado pré-natal é um processo importante que oportuniza avaliação de risco, bem como diagnóstico e tratamento de condições que podem afetar a mulher e seu bebê, sendo capaz de diminuir a morbimortalidade (Carlo \& Travers, 2016).

O governo do estado do Paraná reconhecendo uma estagnação dos indicadores de mortalidade materno-infantil no período de 2006 a 2010, significativos índices de mortes evitáveis, tanto materna (85\%) como infantis (61\%), além de uma realidade com evidente desorganização e disparidade entre as regiões de saúde na oferta dos serviços, implantou no ano de 2012 o Programa Rede Mãe Paranaense (PRMP) como modelo de atenção à saúde materna e infantil no Estado, direcionado aos diferentes níveis de atenção, sendo a Atenção Primária à Saúde (APS) definida como o primeiro contato da usuária com o serviço de saúde e a responsável pelo ordenamento e coordenação do cuidado pré-natal (Paraná, 2018).

Para tanto, lançou-se a Linha Guia ou protocolo de atendimento, pactuações e atribuições em cada nível de atenção, com perspectivas, objetivos estratégicos e indicadores específicos do programa com intuito de acompanhar, monitorar e avaliar as ações do cuidado à mulher e à criança (Paraná, 2018).

A perspectiva da mulher sobre o cuidado recebido durante a gravidez permite identificar se de fato as estratégias do programa, tais como, investimento financeiro, qualificação profissional, implantação do telessaúde, infra-estrutura, consultas e exames, referência e contra-referências à gestante, parturiente e à criança, incentivo ao parto normal respeitando as boas práticas obstétricas e o incentivo ao aleitamento materno está inserido nos serviços de saúde, e, com isso, possibilita a constatação de prováveis inconsistências e capacidade de aperfeiçoamento da assistência à mulher e sua rede familiar (Caldeira et al., 2017). 


\section{Metodologia}

Pesquisa qualitativa fundamentada no referencial da Fenomenologia Social de Alfred Schütz. A abordagem fenomenológica contribui para a compreensão dos fenômenos humanos no cotidiano, a partir de experiências concretas do vivido, permitindo uma análise efetiva sobre as experiências relacionadas ao cuidado pré-natal (Schütz, 2012; Rocha et al., 2018).

O estudo ocorreu nos municípios de Cascavel e Foz do Iguaçu, localizados no extremo Oeste do Estado do Paraná, faixa de fronteira internacional com os países Paraguai e Argentina, local com particularidades relevantes e inerentes as áreas fronteiriças.

Participaram 18 mulheres, acompanhadas na APS destes municípios no período de gravidez e que concluíram todo o cuidado no serviço público de saúde, independente do risco gestacional, quer sejam, Risco Habitual (RH), Risco Intermediário (RI) e Alto Risco (AR) (Paraná, 2018). Aquelas com idade igual ou superior a 18 anos, com facilidade de comunicar-se. A seleção ocorreu por meio de sorteio intencional, em que se priorizaram as mulheres com até seis meses após o parto e procurou-se ampliar a amostra para as diferentes regiões dos municípios.

Todas as recomendações das Resoluções no 466/2012 e nº 510/2016 foram atendidas respeitando-se os critérios para Pesquisa com Seres Humanos e Pesquisa Social (Ministério da Saúde, 2012). Obteve-se parecer favorável do Comitê de Ética da Universidade Estadual de Londrina no 2.053.304 e CAAE 67574517.1.1001.5231 de 09 de maio de 2017.

As entrevistas foram realizadas no período de maio a setembro de 2018. Foi realizado contato por telefone para agendamento do horário e local conforme a disponibilidade da participante. Somente após a Assinatura do Termo de Consentimento Livre e Esclarecido as entrevistas foram iniciadas.

Houve gravação com dispositivo digital e roteiro com questões norteadoras: O que você conhece sobre o Programa Rede Mãe Paranaense? Conte-me sobre a sua gestação e a assistência no pré-natal no serviço de saúde. Você recebeu educação em saúde e orientações dos profissionais durante a gestação? Como você percebe as ações dos profissionais de saúde? Você poderia falar se houveram facilidades e ou dificuldades no acesso ao serviço de saúde durante a gestação? O que você espera do atendimento oferecido pelos profissionais de saúde no que diz respeito ao pré-natal? O que espera dos serviços, das políticas públicas e dos programas voltados à saúde da mulher no que diz respeito ao pré-natal?

Além dos relatos das participantes, a Carteira de Gestante foi fotografada para observação e congruência dos dados. Por fim, para garantir a privacidade, as usuárias foram identificadas pela letra inicial (U) de usuária, seguida por número arábico conforme a ordem das entrevistas.

A análise foi realizada seguindo-se as etapas adotadas por pesquisadores da Fenomenologia Social de Alfred Schütz: transcrição das entrevistas gravadas, leitura minuciosa de cada relato para apreender o sentido íntegro da experiência vivida pelas participantes, agrupamentos de aspectos significativos inclusos nas falas para compor as categorias, análise das categorias com base nas motivações das usuárias, "motivos porque", vivências das mulheres no pré-natal e os "motivos para", expectativas frente aos serviços e profissionais do PRMP (Rocha et al., 2018).

\section{Resultados}

Ao todo 18 mulheres, todas brasileiras, participaram da pesquisa, sendo nove residentes em Cascavel e nove em Foz do Iguaçu. Todas falavam a língua portuguesa, não houve dificuldade na abordagem no que tange à linguagem. A idade das entrevistadas variou de 19 a 38 anos, sendo a média de 26 anos. Dessas, 10 relataram ter união estável, cinco eram casadas e três solteiras; 10 delas declararam serem evangélicas, sete católicas e uma não ter religião. Em relação a escolaridade, seis tinham menos de três anos de estudo. Quanto a profissão, sete mulheres não trabalhavam, cinco trabalhavam sem registro na 
carteira de trabalho e quatro trabalhavam de maneira formal. No que diz respeito ao histórico reprodutivo, 10 já tinham um ou mais filhos e oito eram primigestas.

Em relação ao risco gestacional, cinco foram estratificadas pela equipe de saúde como Risco Habitual (RH), oito de Risco Intermediário (RI) e cinco de Alto Risco (AR). Destas, apenas quatro foram encaminhadas da Atenção Básica para acompanhamento no serviço especializado, sendo três classificadas como AR e uma RI.

A partir dos relatos surgiram duas categorias, uma que evidencia os "motivos porque", ou seja, a vivência no prénatal, constituída por quatro subcategorias: Conhecimento sobre o PRMP; Acesso e acolhimento; Atendimento multiprofissional e suas peculiaridades e Educação em saúde. A última categoria trouxe os "motivos para", as expectativas das mulheres em relação à assistência: O que espera para o cuidado pré-natal no PRMP.

A primeira categoria, Conhecimento sobre o PRMP, trouxe com unanimidade a ausência de conhecimento sobre o programa e sua proposta:

Não, eu não ouvi falar ainda e não faço nem ideia do que seja [...]. (U2)

Nunca ouvi falar [...]. (U6)

Não conheço esse programa [...]. (U10)

No que se refere aos dois pilares do cuidado - o acesso e o acolhimento, as mulheres apontaram algumas dificuldades que encontraram no decorrer do pré-natal:

A primeira fase dos exames foi feita no Posto mesmo. Já a terceira, eu tinha que me locomover lá para outra unidade. Então, pra gente que tá grávida é difícil nesta cidade de fronteira com tudo precário. Tem que pegar o papel do exame em um lugar, em seguida, ir para outro lugar pra fazer, e ainda tem que ir bem cedo, porque en fiquei bastante tempo esperando [...]. (U1)

Eu tive sífilis, então, a doutora me passou o tratamento, e eu precisei ir em outro posto, porque ali não tinha ninguém para aplicar a injeção em mim[...]. (U2)

A gente vai lá consulta e se tem algum encaminhamento para exame eles sempre ligam avisando. Quando eu consultava já deixava marcado para o próximo mês [...]. (U3)

Desde as 33 semanas eu não conseguia marcar meus exames e consulta no posto, a ultrassom do SUS, sem comentários né, até hoje eles não me ligaram para fazer a segunda (risos), tinha 511 pessoas na minha frente [...].

No início é bem complicado para agendar a consulta, que demorava muito, chegava lá assim uma hora da tarde e saía era quatro, cinco horas [...].(U9)

Tive bastante acesso, não tive dificuldade [...]. (U14)

Eu tive dificuldade para agendar consulta com a Dentista, por causa que não tinha mais horário quando eu ia agendar[...]. (U17) 
Em relação às peculiaridades da assistência da assistência, suas falas ressaltaram um acompanhamento pré-natal voltado basicamente para as consultas médicas, sem a participação de outros profissionais de saúde integrantes da equipe.

A Médica não gostei nadinha dela. Meu primeiro pré-natal foi melhor, foi há 7 anos, com outro doutor, não foi aqui, acho que porque também ele era ginecologista, e ela não é, ela é Médica da Família né, então tem uma diferença também[...]. (U5)

Eu ia nas consultas, a Médica fazia as coisas normal, pesava, media a barriga, ouvia o coração, essas coisas, mas nunca ela falou assim como que tem que cuidar do nenê, como que tem que fazer, essas coisas não, eu saía cheia de dúvidas[...]. (U6)

O Médico, às vezes ele fazia a consulta e daí falava que não sabia se era o meu coração ou o coração do bebê, muitas vezes, eles (gestão) colocam assim o clínico geral e ele não entende muito bem do assunto [...]. (U7)

Só tive a primeira consulta com a enfermeira[...]. (U12)

O pré-natal foi somente com o médico mesmo [...]. (U13)

Eu achei que a Médica podia ter explicado melhor, porque ela não me passou muita confiança nas consultas [...]. (U14)

Não era como en esperava, achei muito fraquinho. Porque eu praticamente tinha que obrigar a Médica a me explicar as coisas, ela tinha um pouco de preguiça de explicar as coisas. Com a Enfermeira eu só tive a primeira consulta, para preencher a carteirinha. (U15)

Um dos aspectos destacados pelas participantes foi a ausência de atividades de educação para saúde durante a assistência pré-natal, fato este que as levou a procurar por informações que consideravam importantes junto a familiares e na internet.

Tem muita coisa que a gente precisa ser orientada, né, quando eu descobri que eu estava com essa doença (Sífilis), daí sim, eles me explicaram algumas coisas sobre ela [...]. (U2)

Eu tive mais orientação de colegas [...]. (U3)

Aqui era só aquela consultinha do pré-natal mesmo, mostrar os exames. (U5)

A minha mãe sempre falava e a minha irmã, daí ela sempre falava como que era, me explicava, mas pelo posto mesmo, eu ia mesmo nas consultas com o médico e só [...]. (U6)

Não tive nada no posto não, eu pesquisava alguma coisa na internet, amamentação e tal, até eu não consegui amamentar no peito, eu amamento, mas tem bem pouco leite, tem que dar mamadeira. Isso que eu acho era bem interessante ter no posto, principalmente amamentação né, eu pensei que ia ter alguma palestra, alguma coisa [...]. (U8)

Só na internet (risos), eu ficava pesquisando as coisas na internet, se eu tivesse curiosidade mesmo, eu tinha que perguntar [...]. (U9) 
Os profissionais deviam pelo menos olhar na nossa cara, explicar melhor as coisas, por mais que não seja o primeiro filho, uma gestação é diferente da outra, ele (médico) não me dava atenção [...]. (U18)

A categoria expectativas / sugestões para a assistência pré-natal no PRMP, trouxe às expectativas das usuárias frente ao serviço e profissionais da saúde, as participantes apontaram o desejo de continuar o acompanhamento perto de casa, que este fosse realizado por ginecologista e não clínico geral, que fossem ofertadas reuniões de grupos, palestras e outros serviços de saúde como atendimento odontológico.

Acho que a gente não precisaria sair do nosso Posto, pra ir pra outro fazer os exames [...]. (U1)

Profissionais mais atentos, principalmente o Médico, é difícil esse Médico do Posto, que quer ser um obstetra, sendo que ele não é. O Médico da Família tem uma função, eu aceito ele até como Pediatra e como Clínico Geral, mas como obstetra melhor ele nem tentar porque não dá certo [...]. (U7)

Acho que tem que ter especialista no posto e mais palestra, tipo sobre amamentação, principalmente pra gente que é mãe de primeira viagem, não tem ideia de como que vai ser tudo. E exames mais rápido, porque demorava muito o resultado dos exames [...]. (U8)

Acho que ter grupo de gestação é importante para quem não tem experiência [...]. (U11)

Melhorar os atendimentos médicos. Ofertar mais dentistas que é difícil de conseguir vaga [...]. (U16).

\section{Discussão}

A bagagem de conhecimento, segundo Schütz (2012), adquirida pelas mulheres gestantes sobre o PRMP mostraramse incipientes. Em relação ao acesso e acolhimento, houve dificuldades pelas mulheres durante o pré-natal, quanto à localização geográfica para a realização dos exames, demora nos agendamentos, coleta e resultados, característicos desta região de faixa de fronteira internacional, ainda carente de organização dos serviços públicos de saúde, ao acesso e à qualidade, somando-se ao fluxo de usuários. Observaram-se apontamentos sobre a omissão e a falta de profissionais no serviço. No entanto, algumas mulheres relataram facilidade no acesso e acolhimento de acordo com o preconizado pelo PRMP (Paraná, 2018).

No que tange a omissão e a falta de profissionais nos serviços de saúde de pré-natal, percebe-se que não há reciprocidade de intenções entre a mulher gestante e o profissional que deveria prestar o cuidado, respondendo aos anseios e perspectivas da situação de saúde ou de doença que a gestante busca. A compreensão da ação de cuidar e ser cuidado se dá por meio da reciprocidade de intenções ou de perspectivas (Schütz, 2012).

Acesso e acolhimento são essenciais no cuidado pré-natal, o acesso refere-se à disponibilidade dos serviços, como localização geográfica, horário de funcionamento, sistemas de agendamento, disponibilidade de medicamentos e exames, além do atendimento oportuno. $\mathrm{O}$ acolhimento centra-se individualmente nas gestantes, considerando as preocupações e angústias, permitindo que possam ser ouvidas e integradas em todo o cuidado gravídico-puerperal, permitindo a intersubjetividade na relação social de cuidar e ser cuidado (Rocha et al., 2018). As relações sociais estabelecidas nos espaços de cuidado favorecem a intersubjetividade e a resolutividade na assistência, especialmente no âmbito da APS (Ministério da Saúde, 2012; Vieira et al., 2016).

O mundo cotidiano é o espaço humano com particularidades entre semelhantes, não apenas um mundo físico, mas um mundo social, histórico e cultural (Rocha et al., 2018). A APS traz características territoriais estratégicas por estar fisicamente 
próxima das usuárias, possibilitando vínculo no cuidado, atenção à estratificação de risco, encaminhamento quando necessário a outros níveis de atenção, educação em saúde, cuidado multidisciplinar, garantindo sempre os princípios da humanização (Ministério da Saúde, 2011; Pitilin \& Pelloso, 2017).

No que diz respeito ao atendimento multiprofissional, as mulheres são estimuladas a realizar o cuidado pré-natal durante a gestação como requisito fundamental para uma maternidade saudável e segura. Por isso, o relacionamento entre o profissional e a usuária é de suma importância, reforça o que Schütz (2012) denomina relação face a face, propiciando encontro direto e autêntico. Essa relação oportuniza troca de experiências entre os envolvidos (Marla et al., 2018).

Quanto a dificuldade de acesso na consulta odontológica, apontada nos relatos, vale lembrar que no PRMP, este serviço é pautado como ação indispensável do cuidado pré-natal pelo PRMP, preferencialmente no primeiro trimestre, pois, dentre as mudanças fisiológicas no período da gravidez, existem também várias alterações da cavidade bucal, com um importante número de complicações periodontais com consequências importantes relacionadas ao período gestacional. Diante disto, percebe-se que a gestante possui bagagem de conhecimentos sobre a importância da saúde bucal, porém, entre a equipe multiprofissional e a gestante, parece não haver diálogo e reciprocidade de intenções (Vieira et al., 2016; Baggio et al., 2016).

De acordo com o citado, estudo desenvolvido na $10^{\text {a }}$ Regional de Saúde do Estado do Paraná evidenciou que as consultas odontológicas não acontecem nessa região como propõe o PRMP, o que corrobora com o atendimento no decorrer do pré-natal neste presente estudo (Schütz, 2012; Felczak et al., 2018).

Além da avaliação bucal, a linha de cuidado do PRMP sugere que o acompanhamento pré-natal seja realizado tanto pelo médico quanto pelo enfermeiro. O enfermeiro pode realizar a consulta pré-natal de gestantes com Risco Habitual e acompanhar todos os riscos, quer sejam Habitual, Intermediário e Alto Risco. Esse profissional foi pouco citado pelas mulheres entrevistadas (Paraná, 2018; Magalhães et al., 2018). A situação biográfica da mulher gestante se dá a partir da bagagem de conhecimentos que adquiriu. Essa bagagem de conhecimentos permeia a importância do cuidado pré natal, sobre a equipe de saúde com responsabilidades e ações definidas. Assim, a escassez de conhecimento propiciou invisibilidade do enfermeiro por parte da gestante, instigando maior envolvimento e comprometimento desse profissional.

Em outro contexto estudado sobre as ações do enfermeiro no PRMP, estudo recente mostrou que esses profissionais se preocupam com a qualidade da atenção à saúde da gestante e conhecem os compromissos, indicadores e objetivos do PRMP, exercendo ações de prevenção, cuidado e promoção à saúde. Porém, o estudo contraria o que as mulheres entrevistadas deste presente estudo vivenciaram, ou seja, pouca relação social, pouca interação, pouca reciprocidade de perspectivas ou intenções com esse profissional (Caldeira et al., 2017).

O Enfermeiro é importante integrante da equipe multiprofissional, quer seja no gerenciamento, na liderança de equipes e na assistência. No que tange ao pré-natal, são estabelecidas relações de reciprocidade e relação face a face entre os diversos atores, quer sejam, entre os membros da equipe multiprofissional e, principalmente com a gestante e seus contatos no que tange ao estabelecimento de vínculos dentro das boas práticas obstétricas. Mesmo assim, ainda é necessário difundir a consulta de enfermagem como espaço de cuidado, promoção e prevenção da saúde, independentemente da consulta médica, e incentivar a atuação de enfermeiros no cuidado pré-natal (Caldeira et al., 2017; Felczak et al., 2018).

$\mathrm{Na}$ perspectiva das participantes deste estudo observou-se uma valorização do pré- natal realizado por médico especialista. O acompanhamento pré-natal realizado por médicos generalistas na Atenção Básica conforme o protocolo do PRMP é uma prática segura para as gestantes que apresentam baixo risco gestacional, uma vez que, estudos apontam que não há diferenças no desfecho perinatal no acompanhamento gestacional periódico e rotineiro por obstetras e médicos generalistas. Isto se dá por escassez de bagagem de conhecimentos das gestantes sobre as ações de cada profissional da equipe de saúde (Pitilin \& Pelloso, 2017; Lopes \& Toledo, 2020). 
Além disso, a assistência pré-natal não deve se restringir apenas às ações clínicas- obstétricas deve incluir também as ações educativas na rotina da assistência integral, tecnologias leves capazes de gerar informações relevantes e agregar à sua bagagem de conhecimentos, conceitos para melhor vivenciarem a gestação e maternidade (Lopes \& Toledo, 2020).

Estudo realizado com médicos do PRMP ressaltou a necessidade de capacitação para exercer as ações conforme as propostas do PRMP. A pouca experiência das mulheres com educação em saúde sustenta um relacionamento face a face, pautado pela insegurança, enaltecendo as tecnologias duras, e o desprovimento de cuidados básicos e essenciais para uma maternidade segura e saudável (Schütz, 2012; Lopes \& Toledo, 2020).

O exercício da interdisciplinaridade é essencial no pré-natal e permitem a valorização das necessidades das usuárias, integrando diversos olhares, perspectivas e intersubjetividade, capazes de agregar conhecimento em todo o processo. Neste mesmo pensar, estimular o autocuidado de gestantes e suas famílias durante o pré-natal fortalecer autonomia para todo o ciclo gravídico e puerperal, possibilitando segurança e proteção para a criança que está para nascer (Rocha et al., 2018).

A sustentação da ultrassonografia obstétrica como condição obrigatória para a qualidade do pré-natal, por parte das usuárias, não condiz com o preconizado. A Rede Cegonha indica o exame no primeiro trimestre para verificar a idade gestacional, mas não o considera obrigatório. Enquanto que o PRMP preconiza a ultrassonografia obstétrica no primeiro e segundo trimestre de gravidez (Paraná, 2018; Vieira et al., 2016).

Em relação à educação em saúde, no pré-natal esta é sinônimo de promoção e prevenção à saúde da mulher, soma bagagem de conhecimentos e autonomia para o cuidado de si e do bebê. Por outro lado, também é relevante a educação permanente dos profissionais de saúde. O esclarecimento às usuárias sobre o pré-natal não deve estar baseado apenas em consultas e exames, a integração estratégica que envolve o acolhimento e o reconhecimento das necessidades de saúde, assim como processo educacional, contribui para um cuidado com mais qualidade e menores índices de morbimortalidade (Mayor et al, 2018; Friedrich et al., 2018; Ferreira et al., 2018).

A capacitação dos profissionais da equipe é primordial para que tenham habilidades necessárias e possam atuar de forma recíproca em suas intenções para a criação de vínculo e ações pertinentes ao cuidado pré-natal, podendo minimizar o descontentamento das mulheres relacionado com os exames de ultrassonografia e rejeição do clínico geral. Sabe-se partir da educação em saúde, a gestante pode tornar-se satisfeita parcial ou totalmente, dependendo da abordagem do profissional que a orientada neste processo capaz de esclarecer sobre o ciclo gravídico e puerperal e suas vertentes fisiológicas, psíquicas e sociais, reconhecendo a tecnologia leve associada à interdisciplinaridade como fundamental para o cuidado pré-natal de qualidade e eficaz (Schütz, 2012; Medeiros et al., 2020).

Nesse sentido, a sensibilização e conscientização das equipes de saúde diante da necessidade de aprimorar o processo de comunicação com as usuárias, fazendo com que a educação em saúde se efetive na prática do cuidado pré-natal é extremamente relevante. As evidências científicas apontam a necessidade de melhorar a capacidade técnica e humana das equipes de saúde para trabalhar temas importantes, tais como, o parto, a amamentação e o planejamento familiar, entre outros, potencializando bagagem de conhecimentos sobre temas pertinentes ao ciclo gravídico e puerperal, bem como, ao cuidado com bebê, contribuindo para a diminuição da morbimortalidade materna e infantil (Magalhães et al., 20181; Lopes \&Toledo, 2020; Silva et al., 2020).

O investimento na política de recursos humanos para a qualificação dos profissionais a fim de que adotem modelos assistenciais integrantes, capazes de compreender a importância da educação em saúde para a promoção do autocuidado e autonomia das gestantes, pressupõe uma prática auxiliar na efetivação do cuidado pré-natal eficaz e seguro para a saúde materna e infantil, além da possibilidade de uma experiência bem-sucedida durante a gravidez, o trabalho de parto, o nascimento, o puerpério e o cuidado com o bebê. 
Nesse sentido, um dos caminhos para a autonomia das gestantes é a informação por parte dos profissionais, para que a mulher se sinta segura nas tomadas de decisões, exercendo seu direito de escolha. Assim, faz-se necessário que ações de cuidado ocorram numa perspectiva interdisciplinar com intenções recíprocas no contexto do cuidar-cuidado no pré-natal, considerando-se o atendimento qualificado com profissionais de diversas áreas, especialmente os (as) enfermeiros (as) que tenham habilidades necessárias para oferecer cuidados competentes durante todo o ciclo gravídico e puerparal (Marla et al., 2018; Silva et al., 2020; Félix et al., 2017).

As tipificações, conforme descreve Schütz (2012), encontradas no estudo são específicas deste segmento populacional, estando os resultados limitados a intersubjetivade deste grupo social específico da faixa de fronteira. Há necessidade de pesquisas com um universo amplo para evidenciar a possibilidade de generalização dos achados.

\section{Considerações Finais}

O referencial da Fenomenologia Social de Alfred Schütz permitiu aproximação sobre a vivência das mulheres gestantes que tiveram o cuidado pré-natal por equipe atuante no PRMP. Percebe-se que mesmo com alguns avanços no que se refere a bagagem de conhecimentos das gestantes, bem como, dos profissionais da saúde da APS, ainda há uma lacuna que afeta diretamente a reciprocidade de intenções entre profissionais e mulheres gestantes, dificultando a resolubilidade das necessidades de saúde requeridas. Evidencia-se que ainda há valorização de tecnologias duras e consultas por especialistas, gerando insegurança por parte das mulheres frente a equipe de saúde.

As particularidades da região de faixa de fronteira internacional no que tange a cultura e a linguagem não foram significativas, pois todas as mulheres estudadas eram de nacionalidade brasileira. No que concerne ao acesso aos serviços de saúde, é preciso considerar a demanda e o fluxo de usuários, particularmente de gestantes que buscam os serviços de saúde nesses dois municípios estudados, por ofertarem melhor atendimento à saúde, esta demanda afeta a organização local e, consequentemente a qualidade da assistência.

A efetivação do PRMP por parte das equipes ainda se mostra deficiente na vivência das mulheres nos dois municípios pesquisados, portanto, devem ser reconsideradas algumas formas de atuação das equipes, de modo que trabalhem a perspectiva multidisciplinar e educação em saúde, potencializando a relação face a face, a reciprocidade de intenções e a bagagem de conhecimentos entre os profissionais e a mulher gestante, favorecendo um relacionamento mais ativo e intersubjetivo com qualidade no cuidado pré-natal.

Ao identificar uma assistência com perceptíveis lacunas no cuidado à saúde das mulheres na rede materno-infantil, reconhece-se a necessidade de estudos futuros que alcancem o contexto da assistência pré-natal na percepção das usuárias dos serviços de saúde e tragam, assim, melhorias na qualidade da atenção, podendo contribuir para uma experiência positiva no ciclo gravídico, parto e pós-parto, para todas as mulheres assistidas pelo Sistema Único de Saúde, inclusive em regiões fronteiriças e suas particularidades.

\section{Referências}

Baggio, M. A., Pereira, F. C., Guimarães, A. T. B., Caldeira, S., \& Vieira, C. S. (2016). Programa Rede Mãe Paranaense: análise da atenção pré-natal em uma regional de saúde. Cogitare Enferm, 21(3). http://dx.doi.org/10.5380/ce.v21i3.45301.

Caldeira, S., Luz, M. S., Tacla, M. T. G. M., Machineski, G. G., Silva, R. M. M., \& Ferrari, R. A. P. (2017). Nursing care actions in the paranaense mother network program. Rev Min Enferm, 21(e-992). http://www.dx.doi.org/10.5935/1415-2762.20170002

Carlo, W. A., \& Travers, C. P. (2016). Maternal and neonatal mortality: time to act. J. Pediatr, 92(6), 543-545. https://doi.org/10.1016/j.jped.2016.08.001.

Felczak, C., Ravelli, A. P. X., Skupien, S. V., Ricken, M. H., Bayer, L. D. C. D., \& Almeida, E. A. (2018). Profile of cardiac pregnant women: high-risk. Cogitare Enferm, 23(2). http://dx.doi.org/10.5380/ce.v23i2.49605. 
Félix, R. S., França, D. J. R., Nunes, J. T., Cunha, I. C. B.C., Davim, R. M. B., \& Pereira, J. B. (2017). The nurse in pre-natal care for women in prison system. Rev enferm UFPE, 11(10). 10.5205/reuol.12834-30982-1-SM.1110201731

Ferreira, G. I, Bussadori, J. C. C, Guilhem, D. B., \& Fabbro, M. R. C. (2018). Participation of women in support groups: contributions to the Experience of childbirth. Cienc Cuid Saude, 7(4). https://doi.org/10.4025/cienccuidsaude.v17i4.45138

Friedrich, T. L., Petermann, X. B., Miolo, S. B., \& Pivetta, H. M. F. (2018). Motivações para práticas coletivas na Atenção Básica: percepção de usuários e profissionais. Interface, 22(65). https://doi.org/10.1590/1807-57622016.0833

Gomes, S. P. M., Carvalho, M., Baltazar, M. M. M. (2019). Perfil de estrangeiros e brasileiros atendidos pela odontologia na atenção básica em município de fronteira. Cienc Cuid Saude, 18(2). https://doi.org/10.4025/cienccuidsaude.v18i2.45946

Lopes, P. F., \& Toledo, V. P. (2020). Sentimentos do enfermeiro ao acolher paciente psiquiátrico agitado e agressivo. Rev enferm UFPE, 14(e244485). https://doi.org/10.5205/1981- 8963.2020.244485

Magalhães, S. Q. C, Fracolli, L. A., Siqueira, L. D., Chiesa, A. M., \& Reticena, K. O. (2018). Contributes of pre-natal to self-care of women assisted by family health teams. Cienc Cuid Saude, 17(2). https://doi.org/10.4025/cienccuidsaude.v17i2.39994

Marla, V., Srii, R., Roy, D. K., \& Ajmera, H. (2018). The importance of oral health during pregnancy: a review. Medical Express, 5(mr18002). http://dx.doi.org/10.5935/medicalexpress.2018.mr.002.

Mayor, M. S. S., Herrera, S. D. S. C., Araujo, M.Q., Santos, F. M., Arantes, R. V., \& Oliveira, N. A. (2018). Avaliação dos indicadores da assistência pré-natal em unidade de saúde da família, em um município da Amazônia Legal. Rev Cereus, 10(1). https://dx.doi.org/10.18605/2175-7275/cereus.v10n1p91-100

Medeiros, F. F., Lourenço, J. C., Rodrigues, M. H., \& Ferrari, R. A. P., Serafim, D., \& Cardelli, A. A. M. (2020). Expectativa e satisfação do acompanhamento pré-natal em gestantes de alto risco. REAS/EJCH, 40(e2792). https://doi.org/10.25248/reas.e2792.2020

Mello, F., Victora, C. G., \& Gonçalves, H. Saúde nas fronteiras: análise quantitativa e qualitativa da clientela do centro materno infantil de Foz do Iguaçu, Brasil. (2015). Cienc Saude Colet, 20(7), 2135-45. https://doi.org/10.1590/1413-81232015207.09462014.

Ministério da Saúde (BR). (2012). Resolução nº 466, de 12 de dezembro de 2012: aprova diretriz e normas regulamentadoras de pesquisas envolvendo seres humanos. Conselho Nacional de Saúde.

Ministério da Saúde (BR). (2016). Resolução nº 510, de 07 de abril de 2016: dispõe sobre as normas aplicáveis a pesquisas em Ciências Humanas e Sociais. Conselho Nacional de Saúde.

Ministério da Saúde (BR). (2011). Secretaria de Atenção à Saúde. Departamento de Atenção Básica. Atenção ao pré-natal de baixo risco. Brasília: Ministério da Saúde.

Paraná. (2018). Linha Guia Rede Mãe paranaense. Curitiba, Paraná. (7a ed).

Pitilin, E. B., \& Pelloso, S. M. (2017). Primary care sensitive admissions in pregnant women: associated factors based on the prenatal care process. Texto Contexto Enferm, 26(2). http://dx.doi.org/10.1590/0104-07072017006060015

Rocha, R. R. M., França, A. F. O., Zilly, A., Caldeira, S., Machineski, G. G., \& Silva, R. M.M. (2018). Conhecimento e perspectiva de enfermeiros na rede de atenção materna e infantil do Paraná. Cienc Cuid Saude. 17(1). https://doi.org/10.4025/cienccuidsaude.v17i1.39235

Schütz, A. (2012). Sobre fenomenologia e relações sociais. Vozes.

Silva, I. R., Mendes, I. A. C., \& Ventura, C. A. A. (2020). Strategic directions for strengthening nursing and midwifery: potentialities and connections in the complex perspective. Rev. Latino-Am. Enfermagem, 28(e3380). http://dx.doi.org/10.1590/1518-8345.4456.3380

Vieira, V. C. L., Barreto, M. S., Fernandes, C., \& Scochi, M.J. (2016). Análise da assistência pré-natal em municípios de diferentes portes populacionais do Paraná. Cienc Cuid Saude, 15(1), 125-132. https://doi.org/10.4025/cienccuidsaude.v15i1.16535 